\title{
ESTUDO DAS PARTÍCULAS TOTAIS EM SUSPENSÃO E METAIS ASSOCIADOS EM ÁREAS URBANAS
}

Fabiana Goulart de Carvalho*, André Jablonski

Escola de Engenharia - Universidade Federal do Rio Grande do Sul (UFRGS) - Av. Osvaldo Aranha, 99/513 - 90046-900 Porto Alegre - RS

Elba Calesso Teixeira

Fundação Estadual de Proteção Ambiental (FEPAM) - Rua Carlos Chagas, 55 Centro - 90030-020 - Porto Alegre - RS

Recebido em 30/6/99; aceito em 26/4/00

\begin{abstract}
STUDY OF TOTAL SUSPENDED PARTICLES AND THE ASSOCIATED METALS IN URBAN AREAS. This work aims at the study of the air quality determination regarding the total suspended particles (TSP) and the associated metals in the counties of Charqueadas and Sapucaia do Sul at the state of Rio Grande do Sul. The TSP samples were collected using high volume samplers and the analysis of the metallic elements was accomplished through ICP-AES. The results revealed that the TSP concentrations, found in the two studied regions, have exceded the current air quality patterns established by the Brazilian Legislation. They also revealed high levels of several of the elements being attributed to the presence of anthropogenic sources. The correlation between meteorological data (speed and wind direction) and TSP concentrations were significant and revealed strong influence in particle dispersion.
\end{abstract}

Keywords: air quality; metals; anthropogenic sources.

\section{INTRODUÇÃO}

Em todo o mundo, acentua-se a preocupação do homem com o meio ambiente, pois com a evolução tecnológica e o crescimento industrial, continuam surgindo problemas ambientais que vêm atingindo a saúde humana. Dentro desse contexto, tornase importante e oportuna a avaliação dos poluentes atmosféricos em áreas de grande fluxo populacional.

Em áreas urbanas industrializadas a meteorologia e a topografia da área, em relação a distribuição populacional, são fatores que influenciam significativamente no transporte e dispersão dos poluentes do ar, levando a vários tipos de circulação.

Nas últimas décadas as necessidades energéticas tem sido supridas pelo uso de combustíveis fósseis, os quais contribuem para a poluição do ar pela emissão de diversos poluentes como as partículas totais em suspensão (PTS) e metais-traço. Segundo a Legislação Brasileira (CONAMA, 1992) ${ }^{1}$, existem vários parâmetros de avaliação da qualidade do ar, tais como a determinação da concentração de PTS, $\mathrm{SO}_{2}, \mathrm{CO}, \mathrm{O}_{3}$ e $\mathrm{NO}_{\mathrm{X}}$. A Legislação Brasileira estabeleceu padrões de qualidade do ar para concentrações de PTS para curtos e longos períodos. Para o período de 24 horas (curto) existe o padrão de qualidade do ar primário $\left(240 \mu \mathrm{g} / \mathrm{m}^{3}\right)$, cujas concentrações de poluentes que ultrapassarem esse padrão poderão afetar a saúde da população e o secundário $\left(150 \mu \mathrm{g} / \mathrm{m}^{3}\right)$, no qual as concentrações de poluentes atmosféricos estão abaixo do que se prevê para o mínimo efeito adverso sobre o bem estar da população, assim como o mínimo dano à flora e a fauna. Para longos períodos foram definidas as médias geométricas anuais nos valores de $80 \mu \mathrm{g} /$ $\mathrm{m}^{3}$ (primário) e $60 \mu \mathrm{g} / \mathrm{m}^{3}$ (secundário).

No Brasil os combustíveis fósseis são empregados pelas usinas termoelétricas e siderúrgicas acarretando na emissão de partículas enriquecidas em espécies químicas altamente tóxicas. Essas partículas podem causar sérios problemas ao meio ambiente e ao ser humano por permanecerem durante um longo período na atmosfera e por apresentarem em sua superfície concentrações elevadas de metais como $\mathrm{Ni}, \mathrm{Cr}, \mathrm{Pb}, \mathrm{Cd}, \mathrm{Fe}, \mathrm{Mn}$, etc ${ }^{2,3}$.

*e-mail: fgoulartdecarvalho@yahoo.com.br
No sul do Brasil, particulados tem sido alvo de grande preocupação devido a baixa eficiência dos sistemas de controle para as emissões das partículas mais finas. Os processos que utilizam o carvão (termoelétrica, mineração, etc) e as atividades de siderurgia tem provocado alterações na qualidade do ar em algumas cidades do Estado do Rio Grande do Sul. Dentre estas áreas destaca-se o município de Charqueadas, onde estão localizadas importantes jazidas carboníferas do RS, áreas de mineração de carvão a céu aberto e subterrânea, além de uma siderúrgica e uma termoelétrica em operação, as quais emitem particulados. Destaca-se também o município de Sapucaia do Sul, cuja cidade apresenta como fonte antropogênica fixa a Siderúrgica Riograndense; entretanto a existência da Rodovia Federal BR-116, como principal via de acesso, faz com que predomine a poluição veicular.

Esse estudo tem como objetivo apresentar resultados referentes 'as concentrações de PTS e metais associados do ambiente atmosférico dos municípios de Charqueadas e Sapucaia do Sul, possibilitando melhor avaliar a qualidade do ar em locais que apresentam contribuição de atividades antropogênicas, levando-se em conta a influência das condições meteorológicas.

\section{MATERIAIS E MÉTODOS}

\section{Pontos de Amostragem}

As áreas em estudo envolvem dois municípios localizados nas proximidades de Porto Alegre, que são Charqueadas e Sapucaia do Sul. O município de Charqueadas pertence a Região do Baixo Jacuí, a $60 \mathrm{~km}$ de Porto Alegre e as principais vias de acesso são as Rodovia Federal-BR-290 e Estadual-RS-401. Nesse município a estação de amostragem do ar foi instalada à oeste da Siderúrgica Aços Finos Piratini (AFP) e à sudeste da usina termoelétrica de Charqueadas (ELETROSUL), com coordenadas geográficas $29^{\circ} 57^{\prime} 12,8^{\prime \prime}$ S e $51^{\circ} 37^{\prime} 21,4^{\prime \prime O}$ (Figura 1).

Sapucaia do Sul está localizada na região Metropolitana de Porto Alegre, situada a $18 \mathrm{~km}$ à norte da capital, tendo como principal via de acesso a Rodovia Federal-BR-116. Nesse município o amostrador foi instalado numa rótula a aproximadamente 
$1 \mathrm{Km}$ à leste da BR-116 e 1,7 km à sudeste da Siderúrgica Riograndense, com coordenadas geográficas $29^{\circ} 49^{\prime} 11,4^{\prime \prime} \mathrm{S}$ e $51^{\circ} 09^{\prime} 39,0^{\prime}$ 'O (Figura 2).

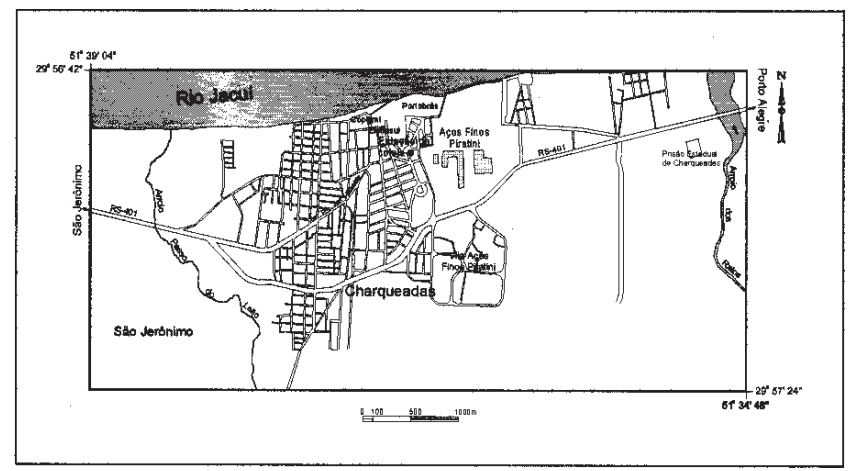

Figura 1. Planta de localização da estação de amostragem em Charqueadas.

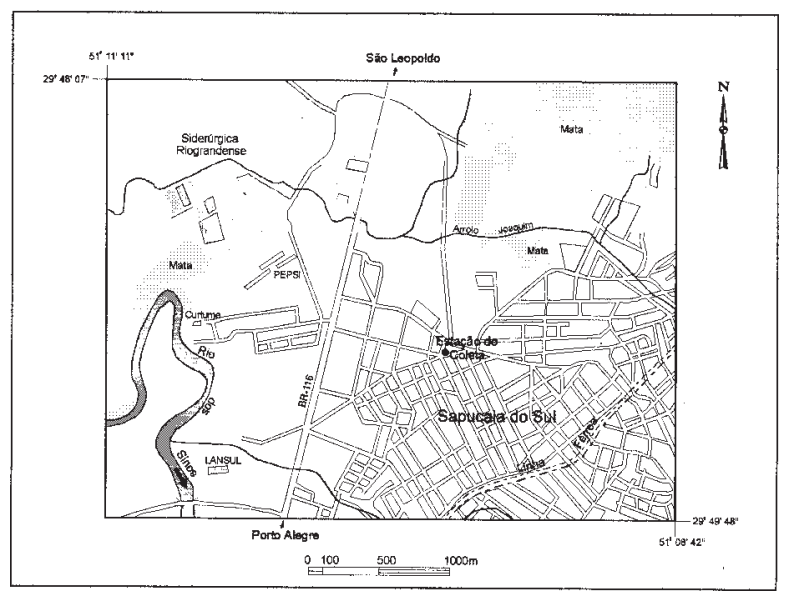

Figura 2. Planta de localização da estação de amostragem em Sapucaia do Sul.

\section{Amostragem e Análise Química}

As amostragens de PTS foram realizadas em filtros de fibra de vidro utilizando amostradores de Grande Volume, do tipo Hi Vol (conforme a norma da ABNT-NBR 9547/86) ${ }^{4}$, no período de maio/ 97 a maio/98, a cada 6 dias por um período de 24 horas.

A concentração de PTS foi obtida determinando-se a massa do material coletado e relacionando com o volume de ar amostrado $\left(\mu \mathrm{g} / \mathrm{m}^{3}\right)$, conforme o método proposto pela EPA ${ }^{5}$. O estudo dos metais foi realizado selecionando apenas os filtros que possuissem maior concentração de PTS, devido ao grande número de amostras coletadas durante o período estudado.
A determinação da concentração dos metais seguiu o método empregado por Teixeira $e t a l^{6}$ que consiste em extrair os metais utilizando $1 / 4$ do papel filtro, contendo PTS com $20 \mathrm{ml}$ de $\mathrm{HNO}_{3}$ concentrado $+20 \mathrm{ml}$ de $\mathrm{H}_{2} \mathrm{O}_{2} 30 \%$, em balão conectado a um condensador de refluxo e aquecido em manta térmica. Posteriormente, eram adicionados $30 \mathrm{ml}$ de $\mathrm{HCl}$ concentrado e $10 \mathrm{ml}$ de $\mathrm{HNO}_{3}$ concentrado, em várias etapas, até a extração final com a eliminação total da matéria orgânica. Para cada amostra eram feitas 2 vias e 2 brancos "matriz" para a determinação de Fe, $\mathrm{Mn}, \mathrm{Cu}, \mathrm{Pb}, \mathrm{Cr}, \mathrm{Ni}, \mathrm{Co}, \mathrm{Cd}$ e $\mathrm{Mg}$ aplicando-se o Espectrômetro de Emissão Atômica por Plasma Indutivamente Acoplado (ICPAES) da marca Jobin-Yvon, tipo $38 \mathrm{~S}$ seqüêncial, com 40,68 MHZ de frequiência e rede de difração dupla (2400-4320 ranhuras). As amostras, previamente digeridas, são introduzidas em plasma de argônio aquecido a $5727^{\circ} \mathrm{C}-7727^{\circ} \mathrm{C}$ por uma fonte de rádio frequiência, permitindo a determinação, simultânea, da concentração de diversos metais-traço, na ordem de ppb, em curto espaço de tempo. As condições de operação do ICP-AES utilizadas na determinação dos metais-traço nos extratos de PTS são apresentadas na Tabela 1 .

Os dados meteorológicos horários de superfície do município de Charqueadas, foram fornecidos pela ELETROSUL situada em Charqueadas. Em Sapucaia do Sul, os dados foram fornecidos pelo Departamento de Proteção ao Vôo do Aeroporto Internacional Salgado Filho de POA-RS. No conjunto de dados estão incluídos a umidade relativa, a precipitação pluviométrica e a direção e velocidade dos ventos. Com relação à direção dos ventos foram consideradas todas as direções, e para cada uma delas foi calculada a frequiência de ocorrência em cada período de amostragem.

\section{RESULTADOS E DISCUSSÃO}

A Figura 3 mostra a variação das concentrações de PTS obtidas no período de maio/97 a maio/98. Esses resultados foram avaliados comparando com os padrões de qualidade do ar primário $\left(240 \mu \mathrm{g} / \mathrm{m}^{3}\right)$ e secundário $\left(150 \mu \mathrm{g} / \mathrm{m}^{3}\right)$ da Legislação Brasileira (CONAMA, 1992) e relacionando com as condições meteorológicas e as fontes locais.

Os resultados mostraram que as duas regiões apresentaram concentrações de PTS elevadas em determinados dias, ultrapassando os padrões de qualidade do ar. Verifica-se que o município de Sapucaia do Sul apresenta maior tendência de poluição por PTS que Charqueadas, atribuindo-se ao fato da estação de amostragem dessa cidade estar situada numa rótula e próxima a Rodovia Federal -BR-116, onde a circulação de veículos automotores é intenso. Em Sapucaia do Sul os níveis de PTS ultrapassaram o padrão primário $\left(240 \mu \mathrm{g} / \mathrm{m}^{3}\right)$ em 9 medições e a concentração máxima aproximou-se de $700 \mu \mathrm{g} /$ $\mathrm{m}^{3}$, ou seja, quase três vezes o padrão primário.

Em Charqueadas os níveis de PTS, de forma geral, atenderam melhor os padrões de qualidade do ar. As concentrações de PTS ultrapassaram o padrão primário em 4 ocasiões, atingindo concentração de até $300 \mu \mathrm{g} / \mathrm{m}^{3}$, atribuindo-se a termoelétrica e as atividades de siderurgia. Nessa mesma figura, observa-se que as concentrações de PTS apresentaram o mesmo comportamento,

Tabela 1. Condições de operação do ICP-AES: comprimentos de onda ( $\lambda$ ) e limites de detecção (L.D).

\begin{tabular}{lcccc}
\hline Condições de operação do ICP-AES & & Elementos & $\lambda(\mathrm{nm})$ & L.D. $\left(\mu \mathrm{g} / \mathrm{m}^{3}\right)$ \\
\hline Força do gerador & $1000 \mathrm{~W}$ & $\mathrm{Cd}$ & 228,802 & 0,001 \\
Vazão do gás plasma & $12 \mathrm{l} / \mathrm{min}$ & $\mathrm{Co}$ & 228,616 & 0,0004 \\
Pressão do nebulizador & $2,6 \mathrm{bar}$ & $\mathrm{Cu}$ & 324,754 & 0,006 \\
Nebulizador utilizado & Meinhardt & $\mathrm{Pb}$ & 220,353 & 0,0008 \\
Vazão de introdução da amostra & $1 \mathrm{ml} / \mathrm{min}$ & $\mathrm{Ni}$ & 231,604 & 0,010 \\
& & $\mathrm{Mn}$ & 257,610 & 0,001 \\
Modo de integração & 2 & $\mathrm{Cr}$ & 267,716 & 0,0002 \\
Fenda & $20 / 25$ & $\mathrm{Fe}$ & 259,940 & 0,042 \\
& & $\mathrm{Mg}$ & 279,553 & 0,001 \\
\hline
\end{tabular}




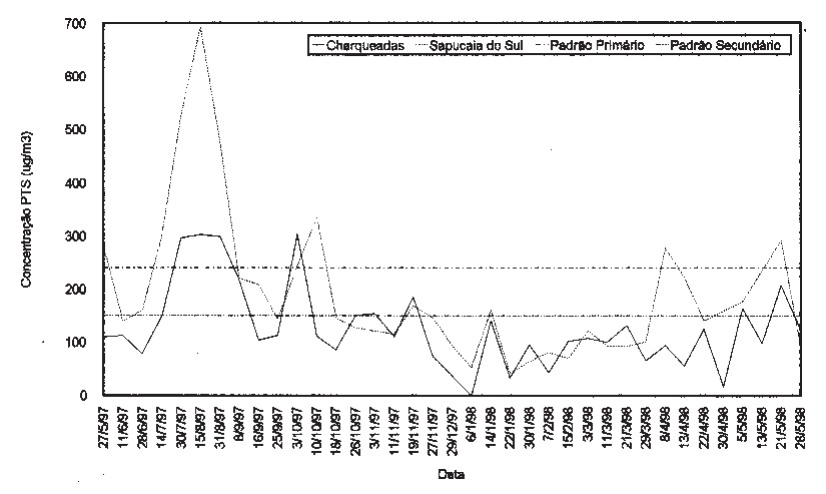

Figura 3. Concentrações de PTS determinadas nas cidades de Charqueadas e Sapucaia do Sul no período de maio/97 a maio/98.

com níveis superiores nos períodos de outono/inverno e decrescendo acentuadamente nos períodos de primavera/verão, indicando forte influência das condições meteorológicas associadas às fontes antropogênicas, na dispersão de PTS.

A fim de examinar a dispersão de PTS, relacionou-se as amostras com concentração superior ao padrão secundário (150 $\mu \mathrm{g} / \mathrm{m}^{3}$ ) com o conjunto de dados meteorológicos (Tabela 2).A partir da Tabela 2, verifica-se que nos períodos de chuva nula ou muito baixa ocorreu, provavelmente, a resuspensão do material particulado, ocasionando concentrações elevadas de PTS. Os ventos na região de Charqueadas foram predominantemente SE/E e com baixa intensidade, sugerindo que as PTS provém principalmente da Siderúrgica Aços Finos Piratini, devido a presença dessa indústra na direção SE do ponto de amostragem. Na região de Sapucaia do Sul, houve predominância de ventos fracos em várias direções (W, NW, E), dificultando a identificação da principal fonte de emissão de PTS.
Nota-se que a direção do vento pode influenciar significativamente no comportamento das concentrações de PTS. Desta forma, a seguir apresentamos, nas Figuras 4 e 5, os dados de concentração de PTS obtidos relacionados com as direções do vento de cada amostragem para melhor caracterizar essa influência.

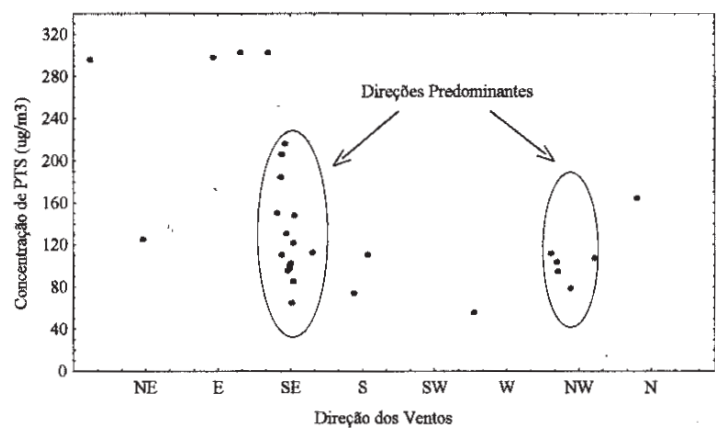

Figura 4. Direção dos ventos para a cidade de Charqueadas.

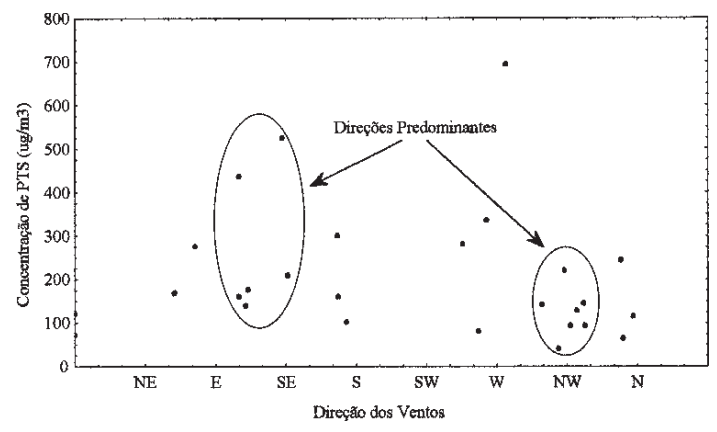

Figura 5. Direção dos ventos para a cidade de Sapucaia do Sul.

Tabela 2. Amostragens com concentrações de PTS superiores ao padrão secundário e dados meteorológicos.

\begin{tabular}{|c|c|c|c|c|c|}
\hline \multirow[b]{2}{*}{$\begin{array}{c}\text { Data } \\
\text { de amostragem }\end{array}$} & \multirow[b]{2}{*}{$\begin{array}{l}\text { Conc. de PTS } \\
\left(\mu \mathrm{g} / \mathrm{m}^{3}\right)\end{array}$} & \multicolumn{2}{|c|}{ Charqueadas } & \multirow[b]{2}{*}{$\begin{array}{l}\text { Velocidade } \\
\text { do vento } \\
(\mathrm{m} / \mathrm{s})\end{array}$} & \multirow[b]{2}{*}{$\begin{array}{c}\text { Umidade } \\
\text { Relativa } \\
(\%)\end{array}$} \\
\hline & & $\begin{array}{l}\text { Precipitação } \\
\text { pluviométrica } \\
(\mathrm{mm})\end{array}$ & $\begin{array}{l}\text { Direção } \\
\text { do Vento }\end{array}$ & & \\
\hline $30 / 07 / 97$ & 295,50 & 0 & $\mathrm{~N}$ & 1,9 & 78,70 \\
\hline $15 / 08 / 97$ & 301,92 & 0,2 & $\mathrm{E} / \mathrm{SE}$ & 1,8 & 77,25 \\
\hline $31 / 08 / 97$ & 298,04 & 0,1 & $\mathrm{E} / \mathrm{NE}$ & 1,3 & 75,18 \\
\hline 08/09/97 & 216,02 & 12,4 & $\mathrm{SE} / \mathrm{E}$ & 1,7 & 76,56 \\
\hline $02 / 10 / 97$ & 302,95 & 0 & $\mathrm{SE} / \mathrm{E}$ & 3,8 & 76,56 \\
\hline 03/11/97 & 153,34 & 0 & SE & 1,9 & 76,00 \\
\hline $19 / 11 / 97$ & 184,50 & 0 & $\mathrm{SE} / \mathrm{E}$ & 2,6 & 75,10 \\
\hline $05 / 05 / 98$ & 163,24 & 0 & NW/SE & 1,5 & 73,16 \\
\hline $21 / 05 / 98$ & 205,94 & 0 & SE/E & 1,7 & 75,00 \\
\hline \multicolumn{6}{|c|}{ Sapucaia do Sul } \\
\hline $27 / 05 / 97$ & 281,00 & 11,10 & SE & 1,6 & 78,4 \\
\hline 28/06/97 & 159,56 & 0 & W & 2,9 & 75,1 \\
\hline $14 / 07 / 97$ & 300,10 & 0 & $\mathrm{~W}$ & 4,7 & 83,1 \\
\hline $30 / 07 / 97$ & 525,60 & 0 & SE & 2,4 & 79,2 \\
\hline $15 / 08 / 97$ & 692,62 & 0 & $\mathrm{~W}$ & 1,9 & 75,3 \\
\hline $31 / 08 / 97$ & 436,79 & 0 & NW & 2,6 & 74,1 \\
\hline 08/09/97 & 219,72 & 1,20 & $\mathrm{E}$ & 3,1 & 83,2 \\
\hline $16 / 09 / 97$ & 207,83 & 7,20 & NW & 2,4 & 68,8 \\
\hline 03/10/97 & 244,07 & 11,30 & $\mathrm{E}$ & 5,4 & 83,7 \\
\hline $10 / 10 / 97$ & 335,62 & 1,90 & W & 2,3 & 75,7 \\
\hline 08/04/98 & 276,51 & 1,80 & $\mathrm{~W}$ & 4,6 & 67,0 \\
\hline $13 / 04 / 98$ & 221,15 & 16,10 & NE & 2,1 & 91,0 \\
\hline $05 / 05 / 98$ & 176,16 & 0 & $\mathrm{E}$ & 1,9 & 77,0 \\
\hline $21 / 05 / 98$ & 290,35 & 0 & $\mathrm{E}$ & 2,5 & 81,0 \\
\hline
\end{tabular}

OBS:. A primeira direção do vento refere-se a predominante e a outra secundária. 
Tabela 3. Concentração dos elementos estudados e PTS $\left(\mu \mathrm{g} / \mathrm{m}^{3}\right)$ nas regiões de Charqueadas e Sapucaia do Sul.

\begin{tabular}{lllllll}
\hline Metais & Mínimo & $\begin{array}{c}\text { Charqueadas } \\
\text { Média }\end{array}$ & Máximo & Mínimo & $\begin{array}{c}\text { Sapucaia do Sul } \\
\text { Média }\end{array}$ & Máximo \\
\hline $\mathrm{Fe}$ & 3,17 & 17,96 & 59,9 & 1,84 & 5,37 & 9,30 \\
$\mathrm{Cd}$ & 0,002 & 0,005 & 0,020 & 0,001 & 0,003 & 0,005 \\
$\mathrm{Co}$ & 0,001 & 0,013 & 0,036 & 0,001 & 0,006 & 0,026 \\
$\mathrm{Cu}$ & 0,114 & 0,212 & 0,409 & 0,284 & 0,476 & 0,950 \\
$\mathrm{~Pb}$ & 0,009 & 0,088 & 0,242 & 0,017 & 0,084 & 0,293 \\
$\mathrm{Ni}$ & 0,019 & 0,172 & 0,706 & 0,005 & 0,018 & 0,036 \\
$\mathrm{Mn}$ & 0,020 & 0,786 & 2,48 & 0,038 & 0,168 & 0,199 \\
$\mathrm{Cr}$ & 0,039 & 0,235 & 0,706 & 0,010 & 0,018 & 0,028 \\
$\mathrm{Mg}$ & 0,224 & 0,883 & 1,77 & 0,522 & 1,09 & 1,60 \\
$\mathrm{PTS}$ & 130,40 & 182,20 & 302,95 & 70,12 & 223,56 & 436,79 \\
\hline
\end{tabular}

Observa-se na Figura 4 a ocorrência significativa de ventos da direção sudeste (SE) e noroeste (NW), indicando influências da Siderúrgica Aços Finos Piratini e da usina termoelétrica, respectivamente. No entanto, em Sapucaia do Sul os ventos apresentaram maior freqüência da direção leste (E), sudeste (SE) e noroeste (NW), sendo que na direção SE encontra-se instalada a Siderúrgica Riograndense, sugerindo contribuição desta indústria na emissão de particulados.

Os dados de metais associados a PTS, encontram-se na Tabela 3, onde são apresentados os valores mínimos, máximos e as médias para os filtros analisados.

Dentre os metais estudados o $\mathrm{Fe}$ foi o elemento que apresentou concentração mais elevada no ambiente para todos os filtros analisados e os níveis deste elemento foram mais significativos no município de Charqueadas. A presença de teores elevados de $\mathrm{Fe}$ e, em parte de $\mathrm{Ni}, \mathrm{Cr}$ e $\mathrm{Mn}$ na região de Charqueadas, associado à direção do vento predominantemente SE, confirma a contribuição da Siderúrgica na contaminação de metais no ambiente. Isto era esperado pelo fato dessa indústria empregar várias matérias-primas contendo $\mathrm{Fe}$ e outros elementos para a produção do aço acarretando na emissão destes metais. Além disso, isto pode ser constatado visualmente, pela emissão de uma intensa fumaça avermelhada na siderúrgica, indicando a presença de óxidos de Fe. A emissão intensa de poluentes pela siderúrgica é atribuída ao fato de que a Siderúrgica AFP não apresenta nenhum sistema de controle para emissões atmosféricas.

Os demais elementos estudados mostraram também concentrações mais significativas no município de Charqueadas, sendo observado que além da siderúrgica, a termoelétrica está contribuindo nas emissões de $\mathrm{Cu}, \mathrm{Pb}, \mathrm{Ni}$ constatado pelas direções dos ventos predominantes SE (siderúrgica) e NW (termoelétrica).

No município de Sapucaia do Sul os níveis de Fe foram também mais elevados que os demais, porém, nesta área não foi possível identificar de forma mais precisa as principais fontes antropogênicas pelo fato do ponto de amostragem estar localizado numa rótula e nas proximidades da BR-116 onde predomina a poluição veicular.

Segundo Steven et al $^{7}$, além do Fe outros elementos estão presentes na composição natural dos combustíveis, e muitos destes ( $\mathrm{Zn}, \mathrm{Cu}, \mathrm{Mg}$, Ca, etc) são utilizados como aditivos da gasolina e do óleo do motor para evitar a oxidação e auxiliar a lubrificação, sendo liberados para a atmosfera através dos gases da exaustão dos veículos. Nessa região os elementos estudados apresentaram concentrações significativas com ventos em direções diversificadas, dificultando a identificação da fonte emissora. Os metais $\mathrm{Cd}$ e $\mathrm{Co}$ apresentaram sistematicamente níveis baixos.

\section{CONCLUSÕES}

A partir dos resultados obtidos, pode-se concluir que as duas regiões encontram-se afetadas por PTS, principalmente em Sapucaia do Sul. As concentrações de PTS excederam os padrões de qualidade do ar estabelecidos pela Legislação Brasileira, apresentando níveis superiores nos meses de outono/inverno.

Quanto aos metais, foram verificadas concentrações elevadas de Fe no ambiente, principalmente em Charqueadas, sugerindo influência da Siderúrgica AFP. Os demais elementos Mn, $\mathrm{Mg}, \mathrm{Cu}, \mathrm{Cr}$ e Ni foram provavelmente originados da siderúrgica local e da usina termoelétrica, uma vez que os ventos foram predominantes nas direções onde encontram-se instaladas estas fontes (SE e NW).

Na região de Sapucaia do Sul, foi constatada influência da Siderúrgica Riograndense e, principalmente dos veículos automotores na emissão dos elementos estudados. Porém, não foi possível determinar a origem da contribuição antropogênica, uma vez que o ponto de amostragem localiza-se numa rótula e nas proximidades da Rodovia Federal BR-116, dificultando na avaliação da principal contribuinte de PTS e, conseqüentemente, dos metais.

\section{REFERÊNCIAS}

1. CONAMA - Conselho Nacional do Meio Ambiente. Resoluções Conama, 1992.

2. Brook, J. R; Dann, T. F.; Burnett, R. T.; J. Air \& Waste Manage. 1997, 47, 2.

3. Smith, I. M.; Trace Elements From Coal Combustion Emissions, London, 1987.

4. Associação Brasileira de Normas Técnicas (ABNT). Determinação da Concentração Total pelo Método do Amostrador de Grande Volume: NBR-9547; 1986.

5. EPA - Quality assurance handbook for air pollution measurements system, ambient air specific methods; Vol. I and II; 1987.

6. Teixeira, E. C.; Avaliação da Poluição Atmosférica; FEPAM, 1998.

7. Steven, H. C.; Mulawa, P. A.; Balli, J.; Donase, C.; Weibel, A.; Sagebiel, J. C.; Environ. Sci. Technol. 1997, 31, 3405 . 